Dementia

and Geriatric

Cognitive Disorders
Dement Geriatr Cogn Disord 2005;20:192-197

DOI: $\underline{10.1159 / 000087301}$
Accepted: March 7, 2005

Published online: August 3, 2005

\title{
Efficacy of Rivastigmine in Alzheimer's Disease Patients with Rapid Disease Progression: Results of a Meta-Analysis
}

\author{
Martin R. Farlow ${ }^{\mathrm{a}}$ Gary W. Small ${ }^{\mathrm{b}}$ Peter Quarg $^{\mathrm{c}}$ Andreas Krause $^{\mathrm{c}}$ \\ a Indiana University School of Medicine, Indianapolis, Ind., ${ }^{b}$ UCLA Neuropsychiatric Institute, \\ Los Angeles, Calif., USA; ${ }^{\mathrm{C}}$ Novartis Pharma AG, Basel, Switzerland
}

\section{Key Words}

Cholinesterase inhibitor $\cdot$ Rivastigmine $\cdot$ Alzheimer's disease $\cdot$ Rapid progression

\begin{abstract}
Background: Alzheimer's disease (AD) patients experiencing more rapid symptom progression are likely to have a poorer prognosis than those experiencing slow symptom progression. In a recent retrospective analysis, treatment effects of rivastigmine were more pronounced in $A D$ patients with rapid cognitive decline than in those with slow cognitive decline. This warranted further investigation. Methods: Rapidly and slowly progressing patients were identified by rates of cognitive decline $[\geq 4$ points and $<4$ points, respectively, on the Alzheimer's Disease Assessment Scale - cognitive subscale (ADAS$\operatorname{cog})$ ] during 26 weeks of placebo treatment in four randomized controlled trials (weeks 0-26). This meta-analysis evaluated rates of cognitive decline in both subgroups during subsequent open-label rivastigmine 26-week extension studies (weeks 26-52). A longitudinal mixed effects model compared cognitive decline in rapidly and slowly progressing patients, including correction for possible regression to the mean. Results: 180 (75\%) rapidly and 337 (78\%) slowly progressing patients provided ADAS-cog data after 26 weeks of open-label rivastigmine
\end{abstract}

treatment. Improvements in cognitive symptoms were observed during the first 12 weeks, which were more pronounced in patients with rapid progression than in those with slow progression. Rapidly progressing patients experienced significantly greater cognitive benefits than slowly progressing patients $(p=0.029)$, who experienced a modest decline in cognitive symptoms at the end of the study. Comment: Patients experiencing rapid symptom progression may receive greater benefit from rivastigmine than those with slow progression. In this study, cholinesterase inhibition appeared to be of particular utility in the management of $A D$ patients whose symptoms were rapidly worsening.

Copyright (C) 2005 S. Karger AG, Basel

Disclosures: Dr. Martin Farlow receives grants from Novartis, Pfizer, Forest Laboratories, Eunoe Inc., Janssen, Eli Lilly and Co., Elan and Pharmanet LLC. Dr. Farlow has acted as a paid consultant for Novartis, Janssen, Eli Lilly and Co., Best Practice, Memory, and Accera Inc., and is on speaker bureaus for Novartis, Forest Research, and Eisai/Pfizer. Dr. Gary Small reports having been a consultant for, having served on advisory boards for, and having received honoraria for lectures from Pfizer, Eisai, Novartis, Lilly, Organon, Forest, Janssen, Abbott, Memory Fitness Institute, Amersham, and CTI. Dr. Peter Quarg and Andreas Krause are employees of Novartis Pharma AG, Basel, Switzerland.

\section{KARGER}

Fax +4161306 1234 E-Mail karger@karger.ch www.karger.com
(C) 2005 S. Karger AG, Basel

$1420-8008 / 05 / 0203-0192 \$ 22.00 / 0$

Accessible online at:

www.karger.com/dem
Dr. Martin R. Farlow

Department of Neurology, Clinical Building, Room 299

541 Clinical Drive, Indiana University School of Medicine

Indianapolis, IN 46202-5111 (USA)

Tel. +1 317274 2893, Fax +1 317278 3930, E-Mail mfarlow@iupui.edu 


\section{Introduction}

Alzheimer's disease (AD) is a progressive neurodegenerative disorder, characterized by the degeneration of cholinergic neurons in the nucleus basalis, and the presence of amyloid plaques and neurofibrillary tangles [1].

The rate of AD progression varies considerably between individuals and is believed to be influenced by a number of factors, including genetic (e.g., apolipoprotein E $\varepsilon 4$ polymorphism or butyrylcholinesterase-K allele), age at onset, gender, duration of illness (disease stage), and the presence or absence of extrapyramidal features [2-9]. It has been shown that patients presenting with a rapid rate of cognitive decline will continue to experience clinically significant symptom progression sooner than those presenting with relatively slow rates of cognitive decline [9]. Furthermore, another study demonstrated that, after adjusting for age, gender and education, mortality in $\mathrm{AD}$ patients was still very strongly associated with their rate of cognitive decline [10]. Therefore, $\mathrm{AD}$ patients experiencing more rapid progression early in the disease need particular attention as they are more likely to reach clinically significant milestones earlier, have a poorer prognosis, and a higher mortality rate than those experiencing slow symptom progression. An assessment of how rapidly the illness is progressing as well as how severely the AD patient is currently affected would, therefore, be important in determining prognosis, a frequent concern of families and/or caregivers.

A recent retrospective analysis of one randomized, placebo-controlled rivastigmine study, and its open-label extension, showed that the treatment response to rivastigmine on measures of cognitive and functional performance during the open-label extension was more pronounced in $\mathrm{AD}$ patients experiencing rapid cognitive decline on placebo during the randomized phase than in those experiencing a slow rate of decline [11]. The results of this analysis suggested that the rate of $\mathrm{AD}$ progression may predict magnitude and/or likelihood of response to cholinesterase inhibitor (ChEI) treatment. The suggestion that a subset of patients may gain particular benefits from rivastigmine appeared to warrant further investigation. Thus, in this follow-up meta-analysis, using pooled data derived from four large, randomized placebo-controlled rivastigmine studies, we evaluated the effects of rivastigmine on the cognitive performance of $\mathrm{AD}$ patients previously on placebo, stratified by their rates of cognitive decline during the preceding randomized phase.

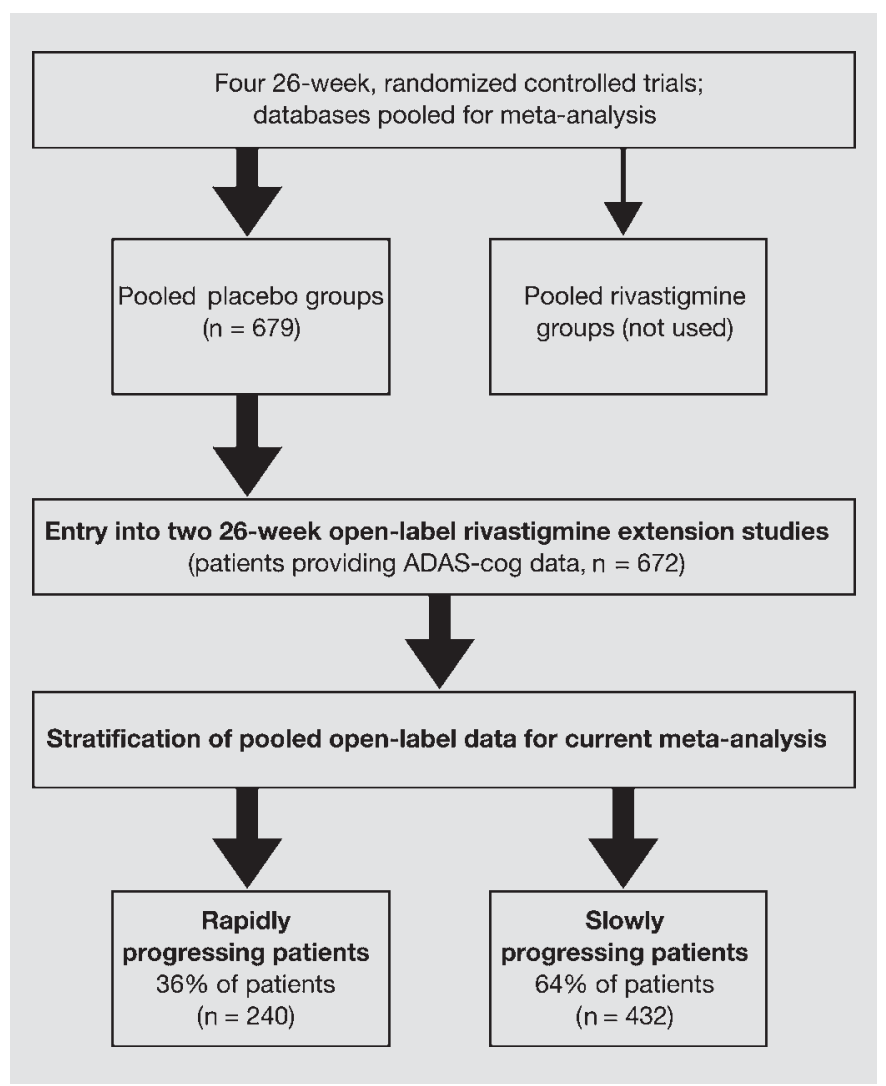

Fig. 1. Current meta-analysis plan.

\section{Methods}

\section{Study Design and Patients}

This meta-analysis utilized data from the first 26 weeks of two large open-label rivastigmine (up to $12 \mathrm{mg}$ daily) extension studies within the ADENA database (fig. 1). Patients entering these studies came from four randomized double-blind, placebo-controlled trials, the full methodologies and findings of which have been previously published [12-15]. Patients with a diagnosis of probable AD were enrolled in these randomized trials according to previously described inclusion and exclusion criteria [12-15] and were randomized to rivastigmine or placebo and treated over a 26-week period.

Informed consent was obtained prior to enrollment and subsequently, after each full year of participation, for those patients remaining on treatment in each open-label extension study. These extension studies were approved by the Ethics Committee overseeing the sites and were performed in accordance with good clinical practice guidelines and the Declaration of Helsinki.

In the open-label extension studies, both investigators and patients remained masked to the patient's original treatment assignment (i.e., placebo or rivastigmine) during the double-blind phase. Patients entering the open-label extension studies were retitrated to their highest well-tolerated rivastigmine dose, starting at $1 \mathrm{mg}$ b.i.d. and rising to a maximum tolerated dose of $6 \mathrm{mg}$ b.i.d. 
Table 1. Baseline (week 26) characteristics of patients entering the open-label extension phase (weeks 26-52), previously identified as rapid or slow progressors while receiving placebo in phase III trials (weeks 0-26)

\begin{tabular}{|c|c|c|c|}
\hline Baseline characteristics & $\begin{array}{l}\text { Rapid progressors } \\
(\mathrm{n}=240)\end{array}$ & $\begin{array}{l}\text { Slow progressors } \\
(\mathrm{n}=432)\end{array}$ & $\mathrm{p}$ value \\
\hline Age, mean $( \pm S D)$, years & $73.7(7.98)$ & $73.0(7.90)$ & 0.262 \\
\hline Male:female, $\%$ & $43: 57$ & $41: 59$ & 0.744 \\
\hline $\begin{array}{l}\text { Dementia duration, mean ( } \pm \mathrm{SD}) \text {, } \\
\text { months }\end{array}$ & $39.8(25.14)$ & $37.7(26.04)$ & 0.311 \\
\hline ADAS-cog score, mean ( \pm SEM) & $34.9(0.8)$ & $20.7(0.5)$ & $<0.001$ \\
\hline \multicolumn{4}{|l|}{ Baseline GDS ratings, $\%$} \\
\hline Very mild & 1 & 3 & \\
\hline Mild & 18 & 37 & \\
\hline Moderate & 40 & 37 & $<0.001$ \\
\hline Moderately severe & 38 & 22 & \\
\hline Severe & 3 & 1 & \\
\hline
\end{tabular}

GDS $=$ Global Deterioration Scale

\section{Cognitive Efficacy Assessment}

In the open-label extension studies, the effect of rivastigmine treatment on cognitive performance was evaluated using the Alzheimer's Disease Assessment Scale - cognitive subscale (ADAS$\operatorname{cog}$ ) [16] and the Mini-Mental State Examination [17].

\section{Current Meta-Analysis}

This meta-analysis focused on the effects of open-label rivastigmine treatment on cognitive decline, as measured on the ADAS-cog [16]. Two groups of patients, identified as those with 'rapid' or 'slow' progression during a 26-week assessment period, while receiving placebo, in the double-blind trials prior to entry into the open-label extension phase, were determined (fig. 1). Patients participating in the rivastigmine arms of the double-blind trials were not included in the two groups (rapid and slow progressors) in this meta-analysis since we hypothesized that therapy may have affected their true rates of cognitive decline over the 26 weeks prior to enrolling in the open-label extension phase. While on placebo, rapidly progressing patients were identified as having experienced a $\geq 4$-point decline in ADAS-cog scores over the previous 26 weeks, while slowly progressing patients exhibited a $<4$-point decline on the ADAS-cog scale during the initial 26-week treatment period.

The ADAS-cog [16] is currently considered the 'gold standard' for assessing changes in cognitive function in AD trials. A higher ADAS-cog score represents poorer cognitive performance, while a negative change from the baseline score with treatment reflects an improvement. Data from ADAS-cog assessments performed at weeks 26 (i.e., start of open-label treatment phase or baseline), 38, 44 and 52 (or at early termination) of the open-label phases of these studies were analyzed.

It could be argued that rapidly progressing patients exhibit greater symptoms and the probability of showing drug effect is higher; therefore, validation of these data was critical. SAS version 8 and S-PLUS version 6.1 were used for summary statistics and modelling, respectively. Statistical comparisons between the rapidly and slowly progressing patients during the open-label phase were made by applying a longitudinal mixed-effects model to assess the change in slope of cognitive symptom progression after initiation of rivastigmine treatment. Importantly, the model adjusted for the baseline differences in cognitive impairment between the slow and rapid progressors. The difference in effect between rapidly and slowly progressing patients was assessed by deviation of the slope for the rapid progressors. In order to correct the respective $p$ value for a possible regression to the mean effect, the data set was simulated 10,000 times from the fitted model with no difference between rapid and slow progressors. Patients were then split into rapid and slow progression subgroups according to the predefined criterion, based on the simulation data. The $\mathrm{p}$ value derived from the empirical data was compared with the distribution of the simulated $p$ values from the null model with no difference in treatment effect (i.e., representing pure chance findings). The resultant $p$ value was corrected for the comparison of the rivastigmine effect on the slowly progressing patients and the rapidly progressing patients. A p value of less than 0.05 was considered to be statistically significant.

\section{Results}

\section{Patients}

In total, 679 patients finished placebo treatment during the double-blind phases of the four rivastigmine clinical trials [12-14] and subsequently entered the open-label phases of one of two extension studies. Six hundred and seventy-two patients provided ADAS-cog data. Of these patients, 240 were 'rapidly progressing' and 432 were 'slowly progressing' while on placebo treatment (i.e., weeks $0-26)$. Of these, 180 (75\%) rapidly progressing patients and 337 (78\%) slowly progressing patients provided ADAS-cog data after 26 weeks of open-label rivastigmine treatment (i.e., weeks 26-52).

The baseline characteristics of these two groups are shown in table 1 . The rapid and slow progression subgroups had similar mean ages and durations of dementia, 
Table 2. Mean ADAS-cog scores of remaining rapid and slow progressors receiving placebo during the double-blind phase of four phase III/IV trials prior to entry into the open-label rivastigmine treatment phase at week 26
Fig. 2. Mean change in ADAS-cog scores from the initiation of open-label rivastigmine (week 26) to the end of the treatment period (week 52) in rapid (O) and slow (○) progressors remaining in the study at each time-point. Standard error bars are included. Asterisks indicate that 3 patients who did not have ADAS-cog data available at week 38 were assessed at later time points.

\begin{tabular}{llllll}
\hline $\begin{array}{lllll}\text { Double-blind placebo } \\
\text { treatment phase, weeks }\end{array}$ & \multicolumn{2}{l}{ Rapid progressors } & & \multicolumn{2}{l}{ Slow progressors } \\
\cline { 2 - 3 } \cline { 5 - 6 } & patients & ADAS-cog score & & patients & ADAS-cog score \\
\hline 0 & 240 & $26.4(0.7)$ & & 432 & $21.5(0.5)$ \\
12 & 237 & $30.3(0.8)$ & & 430 & $20.5(0.5)$ \\
18 & 230 & $32.2(0.8)$ & & 422 & $21.4(0.6)$ \\
26 & 240 & $34.9(0.8)$ & & 432 & $20.7(0.5)$ \\
\hline
\end{tabular}

${ }^{1}$ An increase in ADAS-cog score reflects a worsening of cognitive symptoms. Figures are expressed as mean \pm SEM.

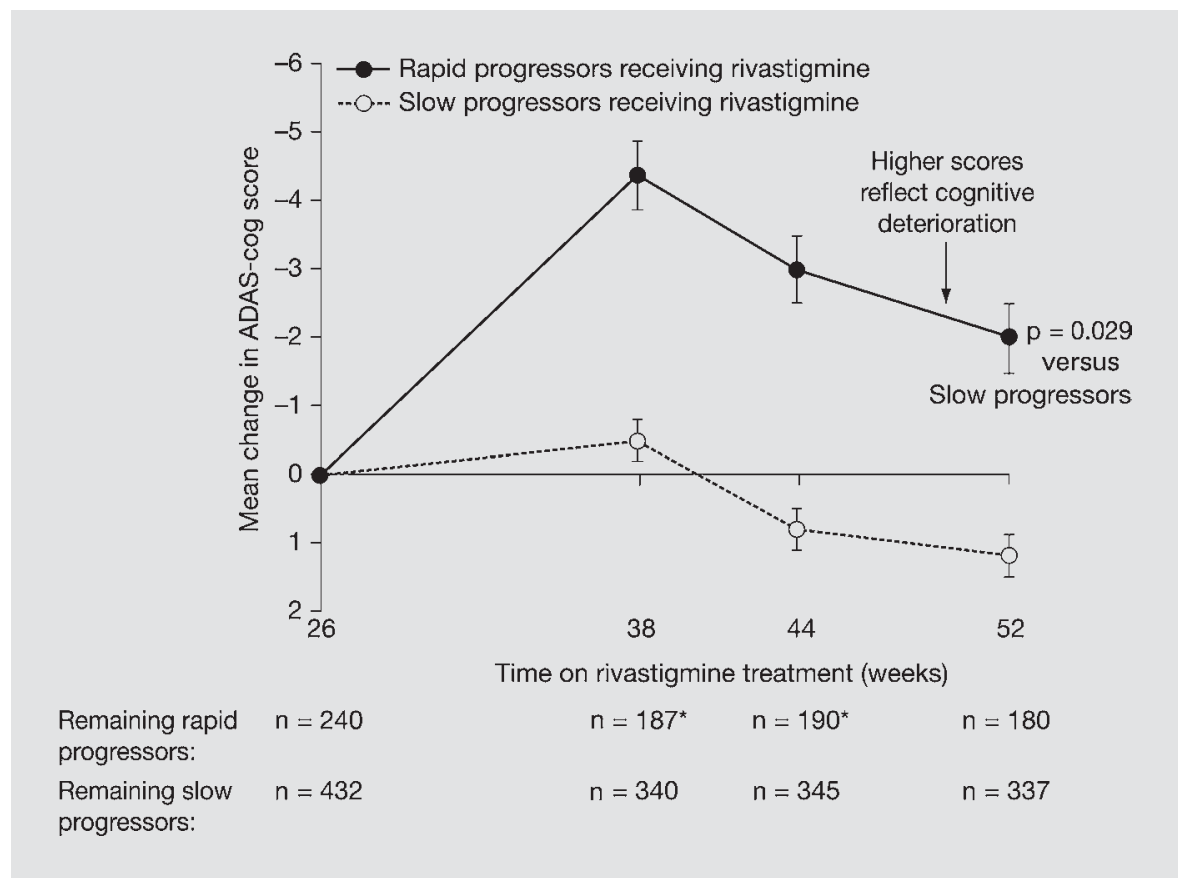

and comprised similar proportions of men and women. As might be expected, rapidly progressing patients had a higher ADAS-cog mean score (34.9 vs. 20.7) at baseline than the slowly progressing patients. Similarly, a greater proportion of rapidly progressing patients had baseline Global Deterioration Scale scores reflecting moderate-severe $\mathrm{AD}$ relative to the slowly progressing patients (81 vs. $60 \%$ ). Rapidly progressing patients reached a mean daily rivastigmine dose of $8.1 \mathrm{mg}$, and slowly progressing patients reached a mean daily dose of $7.9 \mathrm{mg}$.

\section{Assessment of Cognitive Decline}

During 26 weeks of placebo treatment in the doubleblind rivastigmine clinical trials, rapidly progressing pa- tients experienced a worsening of cognitive functioning (mean ADAS-cog increase of 8.5). However, cognitive functioning of slowly progressing patients improved slightly during this 26 -week placebo treatment period (table 2).

Both those with rapid and slow progression treated with rivastigmine experienced improvements in cognitive symptoms (i.e., a negative mean change in ADAS-cog from baseline levels) during the first 12 weeks of the openlabel treatment phase (weeks 26-38). However, rapidly progressing patients receiving rivastigmine experienced a greater magnitude of cognitive improvement than the slowly progressing patients (mean \pm SEM ADAS-cog change: $4.4 \pm 0.5$ vs. $0.5 \pm 0.3$; fig. 2 ). Despite a slight 
decline in ADAS-cog scores (positive mean change increase relative to baseline) from weeks $38-52$, the rapidly progressing patients who remained on treatment for 26 weeks continued to experience an overall improvement in their cognitive symptoms (fig. 2). Furthermore, the improvement in ADAS-cog scores relative to baseline for the patients with rapid progression was significantly greater than that for patients with slow progression $(\mathrm{p}=$ 0.029; fig. 2).

\section{Discussion}

All AD patients remaining on open-label rivastigmine treatment for 26 weeks appeared to experience cognitive benefits, regardless of their prior rate of decline in cognitive functioning. In particular, improvements in cognitive symptoms were observed for patients remaining on rivastigmine treatment after the first 12 weeks of the open-label phase, which were more pronounced in the previously rapidly progressive group than in the slowly progressive group. Overall, rapidly progressing patients remaining on rivastigmine treatment for 26 weeks experienced significantly greater mean magnitude of cognitive benefits than slowly progressing patients.

Another possible explanation for the results seen in this study might be the higher ADAS-cog score of the rapidly progressing patients at the start of open-label treatment. Having considered this, the statistical model used for this meta-analysis adjusted for the baseline differences in cognitive impairment between slow and rapid progressors. Therefore, it is hoped that the influence of baseline cognitive differences between the subgroups will have been minimal. Nevertheless, it is plausible that the psychometric properties of the scale might have allowed the detection of larger changes for individuals who were in the middle of the scale, where maximum rates of change could have been occurring.

These results are consistent with the findings of our previous retrospective analysis of a single rivastigmine trial in which we also found better preserved daily function in patients with rapid progression, as well as improved cognition [11]. To date, rivastigmine is the only ChEI to have demonstrated this differential cognitive effect in AD patients experiencing rapid cognitive deterioration relative to those with slow cognitive deterioration.

Rivastigmine inhibits butyrylcholinesterase (BuChE) in addition to acetylcholinesterase (AChE). There is increasing acceptance that $\mathrm{BuChE}$ has a significant role in cholinergic neurotransmission in the normal brain [18], and this role may increase with advancing severity of $A D$ [19]. Besides its effects on cholinergic neurotransmission, rivastigmine may have broader effects in $\mathrm{AD}$, for example, by affecting cerebral blood flow, amyloidogenesis, and tau phosphorylation [20]. BuChE levels increase in areas of the brain involved in neurodegeneration (i.e., entorhinal and inferotemporal cortex) as AD progresses, while AChE levels decrease [21]. Brain BuChE activity in the temporal cortex has also been correlated with the rate of cognitive decline in patients with dementia [22]. Furthermore, the presence of the BuChE-K variant, which has reduced enzymatic activity, appears to correlate with a slower rate of cognitive decline and preserved attentional performance in patients with $\mathrm{AD}$ and dementia with Lewy bodies [23, 24]. These findings suggest that BuChE may play a role in disease progression and is an important target in addition to $\mathrm{AChE}$ in the treatment of $\mathrm{AD}$, particularly in those patients with a rapid rate of cognitive deterioration.

The findings of the present meta-analysis, together with our previous analysis [11], suggest that patients experiencing rapid symptom progression may be particularly likely to benefit from rivastigmine treatment. Whether similar responses would be seen with other ChEIs has not yet been confirmed.

\section{Acknowledgement}

This research was funded by Novartis Pharma AG. 


\section{References}

1 Rosenstein LD: Differential diagnosis of the major progressive dementias and depression in middle and late adulthood: A summary of the literature of the early 1990s. Neuropsychol Rev 1998;8:109-167.

$\checkmark 2$ Wiebusch H, Poirier J, Sevigny P, Schappert $\mathrm{K}$ : Further evidence for a synergistic association between APOE epsilon 4 and BCHE-K in confirmed Alzheimer's disease. Hum Genet 1999; 104:158-163.

-3 Lehmann DJ, Johnston C, Smith AD: Synergy between the genes for butyrylcholinesterase $\mathrm{K}$ variant and apolipoprotein E4 in late-onset confirmed Alzheimer's disease. Hum Mol Genet 1997;6:1933-1936.

$\checkmark 4$ Panegyres PK, Mamotte CD, Vasikaran SD, Wilton S, Fabian V, Kakulas BA: Butyrylcholinesterase K variant and Alzheimer's disease. J Neurol 1999;246:369-370.

$\checkmark 5$ Selkoe DJ: Alzheimer's disease: Genes, proteins, and therapy. Physiol Rev 2001;81:741766.

-6 Jacobs D, Sano M, Marder K, Bell K, Bylsma F, Lafleche G, et al: Age at onset of Alzheimer's disease: Relation to pattern of cognitive dysfunction and rate of decline. Neurology 1994; 44:1215-1220.

7 Chui H, Lyness S, Sobel E, Schneider L: Extrapyramidal signs and psychiatric symptoms predict faster cognitive decline in Alzheimer's disease. Arch Neurol 1994;51:676-681.

-8 Stern Y, Tang M, Albert M, Brandt J, Jacobs DM, Bell K, et al: Predicting time to nursing home care and death in individuals with Alzheimer disease. JAMA 1997;277:806-812.
9 Doody RS, Massman P, Dunn JK: A method for estimating progression rates in Alzheimer disease. Arch Neurol 2001;58:449-454.

10 Hui JS, Wilson RS, Bennett DA, Bienias JL, Gilley DW, Evans DA: Rate of cognitive decline in Alzheimer's disease. Neurology 2003; 61:1356-1361.

11 Farlow MR, Hake A, Messina J, Hartman R, Veach MS, Anand R: Response of patients with Alzheimer disease to rivastigmine treatment is predicted by the rate of disease progression. Arch Neurol 2001;58:417-422.

12 Corey-Bloom J, Anand R, Veach J: A randomized trial evaluating the efficacy and safety of ENA 713 (rivastigmine tartrate), a new acetylcholinesterase inhibitor, in patients with mild to moderately severe Alzheimer's disease. Int J Geriatr Psychopharmacol 1998;1:55-65.

13 Rösler M, Anand R, Cicin-Sain A, Gauthier S, Agid Y, Dal-Bianco P, et al: Efficacy and safety of rivastigmine in patients with Alzheimer's disease: International randomised controlled trial. BMJ 1999;318:633-640.

14 Schneider A, Anand R, Farlow M: Systematic review of the efficacy of rivastigmine for patients with Alzheimer's disease. Int J Geriatr Psychopharmacol 1998;1:S26-S34.

15 Anand R, Messina J, Hartman R: Dose-response effect of rivastigmine in the treatment of Alzheimer's disease. Int J Geriatr Psychopharmacol 2000;2:68-72.

16 Rosen WG, Mohs RC, Davis KL: A new rating scale for Alzheimer's disease. Am J Psychiatry 1984;141:1356-1364.
17 Folstein MF, Folstein SE, McHugh PR: 'MiniMental State': A practical method for grading the cognitive state of patients for the clinician. J Psychiatr Res 1975;12:189-198.

18 Mesulam M, Guillozet A, Shaw P, Quinn B: Widely spread butyrylcholinesterase can hydrolyze acetylcholine in the normal and Alzheimer brain. Neurobiol Dis 2002;9:88-93.

19 Perry EK, Perry RH, Blessed G, Tomlinson BE: Changes in brain cholinesterases in senile dementia of Alzheimer type. Neuropathol Appl Neurobiol 1978;4:273-277.

20 Ballard CG, Greig NH, Guillozet-Bongaarts AL, Enz A, Darvesh S: Cholinesterases: Roles in the brain during health and disease. Curr Alzheimer Res 2005, in press.

21 Wright CI, Geula C, Mesulam MM: Neurological cholinesterases in the normal brain and in Alzheimer's disease: Relationship to plaques, tangles and patterns on selective vulnerability. Ann Neurol 1993;34:373-384.

22 Perry E, McKeith I, Ballard C: Butyrylcholinesterase and progression of cognitive deficits in dementia with Lewy bodies. Neurology 2003; 60:1852-1853.

23 O'Brien KK, Saxby BK, Ballard GG, Grace J, Harrington F, Ford GA, et al: Regulation of attention and response to therapy in dementia by butyrylcholinesterase. Pharmacogenetics 2003; 13:231-239.

24 Holmes C, Ballard C, Lehman D, David Smith A, Beaumont H, Day IN, Nadeem Khan M, Lovestone S, McCulley M, Morris CM, Munoz DG, O'Brien K, Russ C, Del Ser T, Warden D: Rate of progression of cognitive decline in Alzheimer's disease: effect of butyrylcholinesterase K gene variation. J Neurol Neurosurg Psychiatry 2005;76:640-643. 